\title{
Prevalence and antimicrobial susceptibility of genital Mycoplasmas detected by Mycoplasma IST 2 from urogenital samples in Padua, Italy, between January 2014 and December 2015
}

\author{
Andrea Bartolini, ${ }^{1,2}$ Margherita Scapaticci, ${ }^{2}$ Wassim Akkouche, ${ }^{1}$ Caterina Boldrin, ${ }^{1}$ Lucia Rossi ${ }^{1}$ \\ ${ }^{1}$ Microbiology and Virology Unit, Padua Hospital; ${ }^{2}$ Laboratory Department, San Camillo Hospital, Treviso, Italy
}

\section{Summary}

Background and aims. The aim of this study is to define the epidemiology and the antimicrobial resistance profile of Ureaplasma spp. and Mycoplasma hominis isolated from urogenital specimens of patients attending the Microbiology and Virology Unit of Padua between January 2014 and December 2015.

Materials and methods. The analysis was carried out on a total of 10861 samples. Species identification and antimicrobial susceptibility tests were performed using bioMérieux Mycoplasma IST 2.

Results. 2668 samples (24.6\%) from 2043 patients were positive: 2466 samples positive for Ureaplasma spp. (1897 patients) and 8 samples positive for M. hominis (8 patients), while in 194 samples (138 patients) was detected a coinfection. As for antimicrobial susceptibility of Ureaplasma spp.

\footnotetext{
Correspondence: Andrea Bartolini, San Camillo Hospital, viale Vittorio Veneto 18,33100 , Treviso, Italy.

Tel.: +39.0422.428280/272 - Fax: +39.0422 .428280 .

E-mail: andbarto@libero.it
}

Key words: Genital Mycoplasmas; antibiotic resistance; surveillance.

Contributions: $\mathrm{AB}$ designed the study, performed the laboratory experiments, collected the data, interpreted the findings and wrote the paper. MS interpreted the findings and wrote the paper. WA performed the laboratory experiments and collected the data. CB supervised the laboratory experiments and helped to interpret the findings. LR coordinated the study, supervised the laboratory experiments and helped to interpret the findings.

Conflict of interest: the authors declare no potential conflict of interest.

Received for publication: 20 December 2016.

Revision received: 21 February 2017.

Accepted for publication: 27 February 2017.

(C) Copyright A. Bartolini et al., 2017

Licensee PAGEPress, Italy

Microbiologia Medica 2017; 32:6503

doi:10.4081/mm.2017.6503

This article is distributed under the terms of the Creative Commons Attribution Noncommercial License (by-nc 4.0) which permits any noncommercial use, distribution, and reproduction in any medium, provided the original author(s) and source are credited. we found a resistance $(\mathrm{R}+\mathrm{I})$ rate of $90.6 \%$ for ciprofloxacin, of $74,1 \%$ for ofloxacin, of $52.8 \%$ for azithromycin and of $47.0 \%$ for erythromycin..

Conclusions. Our report shows a high prevalence of Ureaplasma spp. in the study population. Surveillance of antibiotic resistance is critical for an appropriate therapeutic approach, which must always be contextualized with patient's symptomatology.

\section{Introduction}

Ureaplasma spp. and Mycoplasma hominis are common commensals of the urogenital tract of healthy people $(7,11,14,16)$. By contrast they can be frequently involved in human urogenital diseases, such as acute and chronic nongonococcal urethritis, cystitis, pyelonephiritis, vaginitis, cervicitis and chronic pelvic inflammatory disease $(6,16)$. Mycoplasmas can play an important role in the origin of spontaneous abortion, premature rupture of membranes, preterm birth and infertility (12). Furthermore Ureaplasma urealyticum is the bacterium most frequently isolated from the upper genital tract of pregnant women and can cause intrauterine infections (11). The epidemiology of mycoplasmas infection changes in different geographic areas and data reported by authors from various countries are controversial (8). As for the prevalence in Italian population we find in literature several studies such as Tibaldi et al. (13) that found a prevalence of $10.1 \%$ for $U$. urealyticum and $0.1 \%$ for M. hominis in samples collected from pregnant women in Northern Italy, and De Francesco et al. (3) that reported a prevalence of $16.59 \%$ for $U$. urealyticum, $0.2 \%$ for $M$. hominis and $1.84 \%$ of coinfections in patients admitted in Brescia hospital. By contrast other studies reported an high prevalence of infection, for instance Pignatelli et al. (10) found a prevalence of $30.7 \%$ for Ureaplasma spp. isolated from genital tract of childbearingaged women, while Leli et al. (8) reported a prevalence of positive samples for U. urealyticum of $44.5 \%$. Finally Del Gaudio et al. (4) found a prevalence of $30.7 \%$ for Ureaplasma spp. infections in a population of Southern Italy.

M. hominis and Ureaplasma spp. are currently susceptible to agents that interfere with protein synthesis, for example tetracycline, macrolides, aminoglycosides, and chloram- 
phenicol, and to the fluoroquinolones that inhibit topoisomerases $(1,2)$. These bacteria are resistant to beta-lactam antibiotics, including penicillins and cephalosporins, because they lack a cell wall (6) and for the same reason they cannot be seen on Gram stain.

The aim of this study is to define the epidemiology and the antimicrobial resistance profile of Ureaplasma spp. and M. hominis isolated from urogenital specimens of patients attending the Microbiology and Virology Unit of Padua between January 2014 and December 2015.

\section{Materials and methods}

A total of 10861 samples were examined in the clinical laboratory, in detail: 7710 vaginal swabs (71.0\%), 956 urethral swabs (8.8\%), 2055 semen samples (18.9\%) and 140 urine samples $(1.3 \%)$.

The culture, the species identification and antimicrobial susceptibility tests were performed using Mycoplasma IST 2 kit (bioMérieux, Marcy-L'Etoile, France), according to the manufacturer's guidelines. Sensibility test included azithromycin, clarithromycin, erythromycin, josamycin, pristinamycin, doxycycline, tetracycline, ofloxacin and ciprofloxacin. The antimicrobial resistance $(\mathrm{R})$ breakpoints (mg/L) were interpreted according to manufacturer's guide: tetracycline $\mathrm{R} \geq 8$; doxycycline $\mathrm{R} \geq 8$; clarithromycin $\mathrm{R} \geq 4$; azithromycin $\mathrm{R} \geq 4$; erythromycin $\mathrm{R} \geq 4$; josamycin $\mathrm{R} \geq 8$; ciprofloxacin $\mathrm{R} \geq 2$; ofloxacin $\mathrm{R} \geq 4$.

We evaluated antimicrobial susceptibility only in case of bacterial load $>10^{4}$ colony forming units (CFU) per $\mathrm{mL}$. The statistical significance was assessed using $\chi^{2}$ test. A statistically significant difference was accepted when $\mathrm{P}<0.05$.

\section{Results}

Of the 10861 specimens examined, 2668 (24.6\%) from 2043 patients were positive. Among these patients, during the period evaluated, we found that 1653 individuals tested positive in one sample only, 296 patients were found positive in two samples collected in different times, 42 patients in three specimens, 32 patients in four samples, 17 patients tested positive in five samples, two patients were found positive six times and finally from one patient the infection was detected in nine different samples. Among the 2668 positive samples, 2466 were positive for Ureaplasma spp. (1897 patients), eight samples were positive for $M$. hominis (eight patients, load $<10^{4} \mathrm{CFU} / \mathrm{mL}$ ), while in 194 samples from 138 patients a coinfection was detected. In detail $M$. hominis was isolated from eight vaginal swabs and Ureaplasma spp. was isolated from 2251 vaginal swabs collected from 1700 patients aged from 14 to 79 (mean age 32.7 years), 92 urethral swabs collected from 83 patients (43 males aged from 20 to 65, mean age 36.6 years and 40 females aged from 19 to 54, mean age 36.1 years), 103 semen samples from individuals aged from 19 to 61 (mean age 36.6 years) and 20 urine samples collected from 17 patients (one male aged 26 years and 16 females aged from 17 to 45 , mean age 30.8 years). Of the remaining 194 samples with a coinfection, 168 were isolated from vaginal swabs (130 patients), seven from urethral swabs (five males, two females) and one from semen sample.

As for the overall prevalence of genital mycoplasmas, no significant statistical difference $(\mathrm{P}=0.97)$ was found between 2014 (1398 positive samples out of 5694) and 2015 (1270 positive samples out of 5167).

As for antimicrobial susceptibility of Ureaplasma spp. we found a significant resistance $(\mathrm{R}+\mathrm{I})$ rate for ciprofloxacin (90.6\%), ofloxacin (74.1\%), azithromycin (52.8\%) and erythromycin $(47 \%)$, while lower levels were detected for clarithromycin (10\%), josamycin $(5.6 \%)$ and doxycycline (4.4\%). Comparing 2014 to 2015, we found a significant increase of resistance for josamycin, azithromycin, ciprofloxacin and ofloxacin (Table 1).

\section{Discussion and Conclusions}

In this study we found a high prevalence of Ureaplasma spp. isolates $(24.6 \%)$ that was confirmed from Italian epidemiological data of the literature $(4,7,8,10,13)$, as well as the lower rate of samples showing a coinfection (1.79\%) and $M$. hominis infection $(0.07 \%)$.

Table 1. Antimicrobial resistance of Ureaplasma spp.

\begin{tabular}{|c|c|c|c|c|c|c|}
\hline \multirow[t]{2}{*}{ Antibiotic } & \multicolumn{2}{|c|}{2014} & \multicolumn{2}{|c|}{2015} & \multirow[t]{2}{*}{ OR $(95 \%$ CI $)$} & \multirow[t]{2}{*}{$\mathbf{P}$} \\
\hline & $\mathbf{N}$ & R+I & $\mathbf{N}$ & $\mathrm{R}+\mathrm{I}$ & & \\
\hline Doxycycline & 805 & $44(5.5 \%)$ & 718 & $23(3.2 \%)$ & $0.57(0.34-0.96)$ & 0.032 \\
\hline Erythromycin & 818 & 384 (46.9\%) & 718 & 338 (47.1\%) & $1.01(0.82-1.23)$ & - \\
\hline Josamycin & 804 & $35(4.4 \%)$ & 708 & $50(7.1 \%)$ & $1.67(1.07-2.60)$ & 0.022 \\
\hline Azithromycin & 818 & 392 (47.9\%) & 717 & $418(58.3 \%)$ & $1.52(1.24-1.85)$ & $<0.001$ \\
\hline Clarithromycin & 818 & $94(11.5 \%)$ & 718 & $60(8.4 \%)$ & $0.70(0.50-0.99)$ & 0.041 \\
\hline Ciprofloxacin & 818 & $726(88.8 \%)$ & 718 & $666(92.8 \%)$ & $1.62(1.14-2.32)$ & 0.007 \\
\hline Ofloxacin & 814 & $562(69.0 \%)$ & 718 & $573(79.8 \%)$ & $1.77(1.40-2.24)$ & $<0.001$ \\
\hline
\end{tabular}

$\mathrm{N}$, number of susceptibility test evaluated; $\mathrm{R}$, resistant; I, intermediate; $\mathrm{OR}$, odds ratio; $\mathrm{Cl}$, confidence intervals. 
Specifically the general prevalence of Ureaplasma spp. infection found in our study place itself between data reported in other Italian studies such as De Francesco et al. (3) and Pignatelli et al. (10).

Similarly no differences were found regarding susceptibility pattern of the isolates, in fact the literature data showed a good activity of tetracyclines, that were the most active agents against both genital mycoplasmas, followed by macrolides (josamycin, azithromycin, clarithromycin, erythromycin) and fluoroquinolones (ciprofloxacin and ofloxacin) $(3,10,17)$. In our study ciprofloxacin and ofloxacin proved inactive against most of the isolates, azithromycin and erythromycin were inactive for approximately $50 \%$ of the samples, while clarithromycin, josamycin and doxycycline were the most active agents, even if, comparing 2014 to 2015, we found a significant increase of resistance for josamycin, azithromycin, ciprofloxacin and ofloxacin (Table 1). Our results are in accordance with the Italian studies on the antimicrobial susceptibility of Ureaplasma spp. of the past 15 years which showed a high resistance to ciprofloxacin and ofloxacin $(3,4,8,9,10)$ that increase over the years in all Italian regions and a moderate activity of clarithromycin and erythromycin. About the international literature we report the review of Beeton et al. (2) that underline the differences of susceptibility in different countries and also within the same country, such as China, where the rate of resistance to erythromycin varies from $54 \%$ to $1 \%$ in different areas of the country.

Although there is no consensus on the pathological bacterial load to be treated, antibiotic therapy is recommended only in presence of symptoms or in case of growth with a bacterial load $\geq 10^{4} \mathrm{CFU} / \mathrm{mL}$ in order to decrease the development of antibiotic resistance. The spread of antimicrobial resistance compromises the management of infectious diseases with significant implication in clinical practice (2). The excessive use of antimicrobials causes selective pressure for resistance and also the empirical treatment with ineffective antibiotics and poor patient adherence to antibiotic treatment regimens could potentially lead to drug resistance (5).

Although tetracyclines are the most active agents in vitro against both genital mycoplasmas, we advise that the choice of the antimicrobial agent has to be the result of a careful analysis of the susceptibility pattern of each isolate as well as of the antibiotic resistance epidemiology in that specific geographic area.

Considering that literature data reveal substantial differences in antimicrobial susceptibility of genital mycoplasmas isolated from different countries $(2,3,5,15)$, the surveillance of antibiotic resistance is critical for an appropriate therapeutic approach, which must be always contextualized with patients' symptomatology.

\section{References}

1. Bébéar CM, Renaudin H, Charron A, et al. DNA gyrase and topoisomerase IV mutations in clinical isolates of Ureaplasma spp. and Mycoplasma hominis resistant to fluoroquinolones. Antimicrob Agents Chemother 2003; 47:3323-5.

2. Beeton ML, Spiller OB. Antibiotic resistance among Ureaplasma spp. isolates: cause for concern? J Antimicrob Chemother 2017;72:330-7.

3. De Francesco MA, Caracciolo S, Bonfanti C, et al. Incidence and antibiotic susceptibility of Mycoplasma hominis and Ureaplasma urealyticum isolated in Brescia, Italy, over 7 years J Infect Chemother 2013;19:621-7.

4. Del Gaudio T, Distasi MA, Porzio M, et al. Isolation and antibiotic resistance of Ureaplasma spp. isolated from urogenital specimen between 2002 to 2007. Microbiol Med 2009;24:40-42.

5.European Centre for Disease Prevention and Control. Antimicrobial resistance surveillance in Europe 2014. Annual Report of the European Antimicrobial Resistance Surveillance Network (EARS-Net). Stockholm: ECDC; 2015.

6. Gwee A, Curtis N. Ureaplasma: are you sitting comfortably? J Infect 2014;68:S19-23.

7. Kacerovsky M, Pliskova L, Bolehovska R, et al. The impact of the microbial load of genital mycoplasmas and gestational age on the intensity of intraamniotic inflammation. Am J Obstet Gynecol 2012;206:e1-8.

8. Leli C, Mencacci A, Bombaci JC, et al. Prevalence and antimicrobial susceptibility of Ureaplasma urealyticum and Mycoplasma hominis in a population of Italian and immigrant outpatients. Infez Med 2012;20:82-7.

9. Messano GA, Cancrini F, Marsella LT. Antibiotic resistance and defensive medicine, a modern challenge for an old problem: the case of Ureaplasma urealyticum. Ig Sanità Pubbl 2014;70:295-304.

10. Pignatelli S, Pulcrano G, Iula VD, et al. In vitro antimicrobial profile of Ureaplasma urealyticum from genital tract of childbearing-aged women in Northern and Southern Italy. APMIS 2013;122:552-5.

11. Rautava S, Luoto R, Salminen S, et al. Microbial contact during pregnancy, intestinal colonization and human disease. Nat Rev Gastroenterol Hepatol 2012;9:565-76.

12. Taylor-Robinson D. The role of mycoplasmas in pregnancy outcome. Best Pract Res Clin Obstet Gynaecol 2007;21:425-38.

13. Tibaldi C, Cappello N, Latino MA, et al. Maternal risk factors for abnormal vaginal flora during pregnancy. Int $\mathrm{J}$ Gynaecol Obst 2016;133:89-93.

14. Viscardi RM. Ureaplasma species: role in diseases of prematurity. Clin Perinatol 2010;37:393-409.

15. Waites KB, Katz B, Schelonka RL. Mycoplasmas and ureaplasmas as neonatal pathogens. Clin Microbiol Rev 2005; 18:757-89.

16. Waites KB, Schelonka RL, Xiao L, et al. Congenital and opportunistic infections: Ureaplasma species and Mycoplasma hominis. Semin Fetal Neonatal Med 2009;14:190-9.

17. Xiao L, Crabb DM, Duffy LB, et al. Chromosomal mutations responsible for fluoroquinolone resistance in Ureaplasma species in the United States. Antimicrob Agents Chemother 2012;56:2780-3. 